\title{
Encoding origin of the terminal asymmetry effect in recall
}

\author{
GREGORY V. JONES \\ University of Oxford, Oxford, England $O X 13 U D$
}

\begin{abstract}
Jones (1976) found that the accuracy of recall of the order in which a series of pictures was seen was dependent upon the way in which recall was cued. For the latter part of a series, sequential position was a more effective cue for the recall of the corresponding picture than the picture was for sequential position. Two experiments reported here (testing altogether 100 subjects) investigated whether this terminal asymmetry effect (TAE) arises at encoding or only at retrieval. It was found that the TAE was affected by the conditions of presentation of the series but not by the postpresentation factors studied.
\end{abstract}

When elements corresponding to two or more features of a situation are stored in memory, then any of these features when subsequently provided as a cue may be successful in inducing recall of the remainder. For any particular pair of features, $A$ and B, experimental measurement can be made of the probabilities of success in two ways: recall of $B$ when cued by $A$, and recall of A when cued by B. Symmetrical recall of twocomponent visual drawings has been reported by Asch and Ebenholtz (1962), who proposed a principle of associative symmetry to account for their results. The principle states that mental associations are bidirectional, or, equivalently, that it is not possible to form a forward association between $\mathrm{A}$ and $\mathrm{B}$ without simultaneously forming a backward association of equal strength between B and A. Jones (1976) examined recall of sets of photographs of different objects, and also found symmetrical relations in memory between the three visual attributes of color, location, and object type. The visual data were shown to be in quantitative agreement with the hypothesis that the pictures gave rise in memory to what were termed fragments. The fragment (or fragments) deriving from a picture corresponds to a particular subset of its attributes: The provision of one of these attributes as cue renders all the other components of the fragment available for recall. The hypothesis provides an accurate representation of the quantitative relationships between probabilities of recall using different types of single and multiple cues for the recall of a range of materials (see Jones, in press). Binary associative symmetry follows from the hypothesis because recall of $\mathbf{A}$ given $B$ occurs if and only if the relevant fragment contains both $A$ and $B$, in which case cuing with $B$ must conversely make A available for recall.

Requests for reprints should be sent to Gregory V. Jones, University of Oxford, Department of Experimental Psychology, South Parks Road, Oxford, England OX1 3UD.
In the light of these findings, cases in which overt relationships are asymmetric are invested with special theoretical interest as exceptional phenomena. Possible theoretical explanations of asymmetry are considered here in relation to experimental results concerning a particular asymmetric phenomenon, the terminal asymmetry effect.

\section{The Terminal Asymmetry Effect}

As noted above, Jones (1976) found that the relationships in recall between the color, location, and object type of an object shown in a series of pictures were symmetrical. However, in that study there was also a fourth independent attribute, the sequential position of each picture within its presentation set of nine items. Thus, sequential position (first, second, ..., ninth) could either be used as the cue for the other attributes $(\mathrm{seq}=\mathrm{cue})$ or it could itself be recalled by the use of another attribute as cue (seq = answer). It was found that the relationships in recall between sequential position and the three visual attributes were asymmetric, with recall being higher overall when sequential position acted as cue than when it was recalled as answer in each of the three cases. The level of performance for both seq = cue and seq = answer varied considerably over the different values of sequential position, the course of the variation being similar for the three different types of visual attribute. The advantage of seq = cue over seq = answer occurred over the latter half of the list and it is thus referred to in brief as the terminal asymmetry effect (TAE). Several possible theoretical explanations of the effect are considered below. The major question to be considered is whether the effect arises at encoding or only at retrieval.

\section{Encoding Explanation}

Retrieval asymmetry is by definition a necessary factor for the TAE to be observed-nominally identical 
information concerning the end of a list is better retrieved when $s e q=$ cue than when $s e q=$ answer. However, it is possible that retrieval asymmetry per se does not provide a sufficient account. Instead, the retrieval asymmetry may be the consequence of an encoding asymmetry. It is noted below that there is at least one specific form of the general encoding hypothesis which is consistent with the occurrence of the TAE (dual-component hypothesis), although another previously proposed encoding hypothesis does not account for the effect (distinct-associations hypothesis).

Dualcomponent hypothesis. This hypothesis proposes that the memory trace derived from a list item can contain two independent components each representing a different displacement, the distance of an item from either the beginning $\left(\mathrm{s}_{\mathrm{b}}\right)$ or the end $\left(\mathrm{s}_{\mathrm{e}}\right)$ of its list. The accuracy with which these displacements are encoded in memory is postulated to decrease with increasing magnitude in Weberian/Fechnerian manner. Information about the end of the list, for example, is conveyed accurately by the $s_{\mathrm{e}}$ component. At recall, the subject can use either the $\mathrm{s}_{\mathrm{b}}$ or the $\mathrm{s}_{\mathrm{e}}$ information as a basis on which to answer. However, the bulk of his previous experience (e.g., in counting) biases him toward using the $s_{b}$ (forward displacement) rather than the $\mathrm{s}_{\mathrm{e}}$ (backward displacement) information. This bias can be overcome when the subject knows in advance of recall that the relevant item occurred near the end of the list (i.e., when high values of sequential position are used as cues), since the end of the list can be seen to be the more appropriate anchor point (cf. Johnson, 1955, Chapter 10). This use of the $s_{e}$ information produces the advantage of the seq = cue condition in the terminal positions.

Distinct-associations hypothesis. It has frequently been proposed (e.g., Lowry \& Wollen, 1969; Wolford, 1971) that mental associations are formed separately in opposite directions. Asymmetry in the recall of paired associates, for example, is attributed to forward associations being stronger or more readily formed than backward associations. The hypothesis does not appear to offer a satisfactory explanation of the TAE, however, because it seems difficult to provide an additional explanation of why associations from sequential positions to pictures should be stronger than the reverse associations only for higher values of sequential position.

\section{Retrieval Explanation}

This category of explanation lays emphasis upon the processes occurring at the time of recall rather than those at the time of presentation. Asymmetry is postulated to arise from differences in the retrieval of a single type of stored information, rather than from the retrieval of two different types of stored information. One specific example of a retrieval explanation which is consistent with the observation of a TAE by Jones
(1976) is described here (dual-access hypothesis), together with two previously proposed retrieval hypotheses which do not account for this observation (difference in availability or in concreteness hypotheses).

Dual-access hypothesis. This hypothesis is similar to the dual-component hypothesis in proposing that the TAE results from subjects making psychophysical judgments concerning displacements from either the start or the end of the list. It postulates, however, that only one type of information representing sequential position is encoded (this representation may be of constant or of variable accuracy with respect to different values of sequential position). Asymmetry arises because of the different ways in which this information is accessed. When sequential position is provided as cue, it can be translated into information concerning the displacement of the item from either the beginning or the end of the list (whichever is the nearer) and thus acts as a more accurate retrieval cue at the extremities of the list. This hypothesis is related to that suggested by Bjork and Whitten (1974) to account for recency effects in long-term recall, namely, that the temporal distance of an item from the point of recall may be used as an implicit retrieval cue. When, on the other hand, sequential position is itself cued for, it is postulated that the subject's previous experience biases him toward translating the retrieved information into a displacement relative to the start of the list only.

Difference in availability. It has been suggested (Asch \& Ebenholtz, 1962) that asymmetry in recall may result from elements having different availabilities. The availability of an item has been defined as its ease of recall (Asch \& Ebenholtz, 1962), or the readiness with which a verbal unit comes to mind as a label for experience (Horowitz, Norman, \& Day, 1966), with the recall of items of high availability by cues of low availability being more successful than the reverse. Differential availability of pictorial attributes and of sequential position cannot explain the TAE, however, because in Jones' (1976) experiment the subjects were provided at recall with the nine possible values of each of the four attributes, thus, presumably equalizing their availabilities. Furthermore, even if availabilities were not equalized by this procedure, it might be expected that any residual asymmetry in availability would be in favor of the ordinal positions (first, second, ..., ninth) rather than the visual attributes, which would give rise to a TAE opposite in direction to that observed.

Difference in concreteness. Lockhart (1969) presented adjectives and nouns in pairs and found the concrete nouns subsequently acted as better cues for the adjectives than the adjectives did for the nouns, but that abstract nouns produced symmetrical results. Peterson (1971) confirmed the importance of concreteness rather than grammatical class per se. Lockhart suggested that, in general, the greater a cue's 
concreteness, the more effective are the retrieval cues which it provides for the recall of an associated item. This hypothesis, however, cannot account for the TAE since it, also, incorrectly implies that the pictorial attributes should be more successful cues than the more abstract one of ordinal position.

\section{EXPERIMENT 1}

It was shown above that specific forms of both the encoding and retrieval hypotheses appeared to offer satisfactory explanations for the TAE. These two general types of hypothesis differ in their experimental predictions. The encoding and retrieval hypotheses predict that the conditions of presentation and of recall, respectively, should be crucial. The present experiment assessed the effect upon the magnitude of the TAE of variations in the speed of presentation and of recall of series of words. The effect of variation in the rate of recall is of particular interest because it has not been widely studied previously, though Reed (1973) has found that performance in a recognition memory experiment improved as the time allowed for retrieval was increased up to $2 \mathrm{sec}$ (see also Wickelgren, 1976).

The present experiment differs in several respects from that of Jones (1976), most obviously in that the items whose order was to be remembered consisted of words instead of multicomponent pictures. Since the subjects thus had fewer independent attributes to remember, the rates of presentation of the list which were used were increased from one every $2.2 \mathrm{sec}$ (Jones, 1976) to one every $.5 \mathrm{sec}$ or $2 \mathrm{sec}$. Recall was paced instead of unpaced. A pilot study established $5 \mathrm{sec} /$ item as approximately the fastest rate that subjects could manage (each question was on a separate page of a booklet), while a second, slower rate of $8 \mathrm{sec} /$ item was reported by subjects to allow adequate time for thought. As in the experiment of Jones (1976), the complete set of possible responses was printed on each page of the answer booklets; thus, the subject's task in both experiments could be described as one of recognition rather than recall. The experiments differed, however, in that in the previous experiment the complete set of possible cues also was printed on each page of the answer booklets (with the relevant cue in each case having been marked by the experimenter), whereas in the present experiment only the single relevant cue was printed on each page.

\section{Method}

Subjects. The subjects of this experiment were 80 members of the Oxford long-term subject panel. They were between 18 and 30 years of age, and were paid for participating.

Stimuli. Subjects were shown 20 separate sets of 10 words each to remember. The 200 words were randomly selected from those words tabulated by Paivio, Yuille, and Madigan (1968) which were between three and eight letters in length and which had frequencies between 10 and 50 per million in the Thorndike-Lorge word count. The average imagibility (I score) of the words was 5.38. In order to minimize the effects of chance variation in the ease of recall of individual words, the 20 by 10 array was arranged so that there was little variation in the average I score over either the 10 serial positions $(\mathrm{SD}=.09)$ or the 20 different sets $(\mathrm{SD}=.16)$.

Half of the subjects viewed the words at the fast presentation rate of one per $.50 \mathrm{sec}(.45-\mathrm{sec}$ exposure, .05 -sec interval) and half at the slow rate of one per $2.00 \mathrm{sec}(1.75-\mathrm{sec}$ exposure, .25 -sec interval).

Questions. After a delay, the recall of each set of words was tested using answer booklets containing one question per page. Each page showed either a particular ordinal position together with the 10 words of the list (in randomized order) or a particular word of the list together with the 10 ordinal positions 1 st to 10th. The subject had to underline the corresponding word or ordinal position as appropriate.

There were five questions for each set, with the two types of question arranged alternately. Summed over the 20 sets, each of the values of seq $=$ cue and seq $=$ answer occurred five times, once in each of the five question serial positions. Two different sets of questions were used, the second set of questions being comprised of the correct answers to the first set of questions. For each condition, equal numbers of subjects received the two different question sets, insuring that, summed over subjects, the events to be recalled for seq = cue and for seq $=$ answer were the same.

Procedure. Each subject was given a set of instructions fully informative of the nature of the task; in addition, the experimenter paraphrased them informally.

The words of each list were videotaped from computergenerated displays on a Tektronix 604 monitor. "Get ready" was followed by 10 words at either the fast or slow presentation rate, followed after $.25 \mathrm{sec}$ by a three-figure number (e.g., 461), which was displayed for $25 \mathrm{sec}$. Subjects counted aloud backward by threes from this number until it disappeared, when they commenced recall. The delay interval averted a relatively large difference in time between presentation and recall of early and late items in the list. Counting backward by threes (Peterson \& Peterson, 1959) was used also by Jones (1976) and obviated the possibly distorting effects of preferential rehearsal. When the three-figure number disappeared from the screen, an auditory bleep sounded simultaneously. This signaled that the subject should turn over to the next page of the booklet and answer the question it contained. Five more bleeps sounded at either 5 -sec intervals (fast recall rate) or 8-sec intervals (slow recall rate) to inform subjects of the time allowed for each question. Equal numbers of subjects were in each of the four experimental conditions (e.g., fast-slow: fast presentation followed by slow recall).

\section{Results}

Correct recall. Figure 1 shows the variation in the level of correct recall in this experiment. Inspection shows that the TAE occurred only after fast presentation, and that the rate of recall exerted no systematic effect.

The results of a split-plot ANOVA (with presentation rate and recall rate varying between subjects and type of cue and sequential position within subjects) supported this interpretation. The analysis was carried out upon arc-sine transformations of the proportions correct averaged over the first five and the last five sequential positions. In accordance with the above description, the three-way interaction of presentation rate, type of cue, and sequential position was significant $[F(1,76)=8.06, p<.01]$. The only other significant 


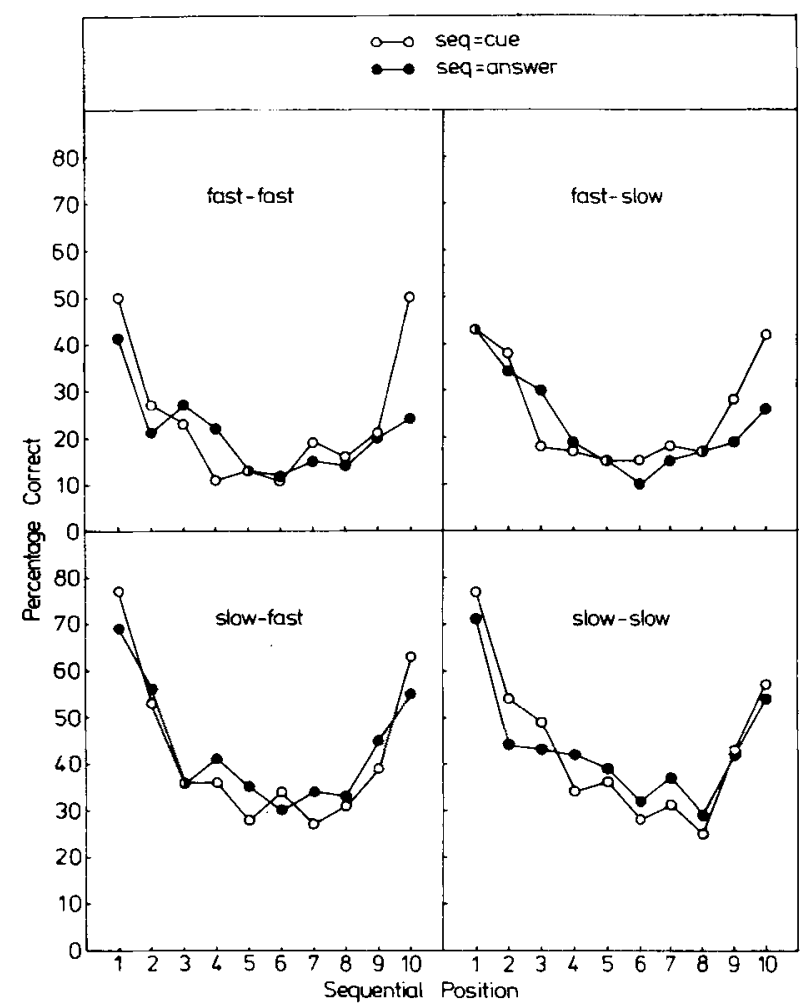

Figure 1. Percentage correct recall in Experiment 1 when sequential position was cue (seq = cue) or answer (seq = answer), for different rates of presentation (first) and of recall (second).

factors were the main effects of presentation rate and of sequential position $[\mathrm{F}(1,76)=43.68, \mathrm{p}<.001$, and $F(1,76)=59.16, p<.001$, respectively]

Figure 1 shows that for both fast-fast and fast-slow conditions, the largest difference between the seq = cue and the seq $=$ answer performance occurred at the last sequential position. For the immediately previous sequential positions, however, asymmetry was masked by the effects of random fluctuation. To obtain reliable estimates of the size of the TAE, it was decided to sum the total asymmetry over the second half of the lists (as in the ANOVA). For the fast-fast condition, the asymmetry totaled 32 percentile points, while for the fast-slow condition it totaled 33 percentile points, showing that the overall size of the TAE remained approximately constant irrespective of the recall conditions.

Centrality. The restriction of the response set in each case to the 10 possible answers meant that each answer corresponded to a particular presentation sequential position (for seq $=$ answer this was the answer itself; for seq = cue it was the presentation sequential position of the word produced as answer). This makes it possible to examine the distribution of answers at each sequential position. Measures obtained from these distributions will be partially independent of the probability correct and, thus, additionally informative.
The me an was chosen as a measure of centrality because of skewness in the distributions for extreme sequential positions.

Figure 2 shows that for fast but not for slow presentation the medians as well as proportions correct display TAEs which furthermore do not appear to be systematically dependent upon the rate of recall. As pointed out by Jones (1976), if the seq = answer data reflect subjects' judgments of the duration of the list up to the cue word, then the median recalled values may be expected to be related to the correct values by a power function, $J(n)=A n^{B}$, where $J(n)$ is the median answer at sequential position $n$, and $A$ and $B$ are constants (Stevens, 1971). In the present experiment the validity of this relationship was not tested for the slow presentation data, since it is clear by inspection that the seq = answer curves in these cases approximate the degenerate equation $J(n)=n$. The application of the equation to the fast presentation data also has two theoretical obstacles. First, the experiment differed from the usual magnitude estimation procedure by employing only a finite response set. Second, the enhanced recall of the last items in the fast presentation conditions indicates some reference to the end rather than the beginning of the lists. Empirically, however, these two factors did not significantly impair the validity of the power relationship. Log-log regression analysis showed that the function accounted for $95.4 \%$ and 98.3\% of the variance in the fast-fast and fast-slow conditions, respectively, with the exponent $B$ taking the values of .44 and .55 , respectively.

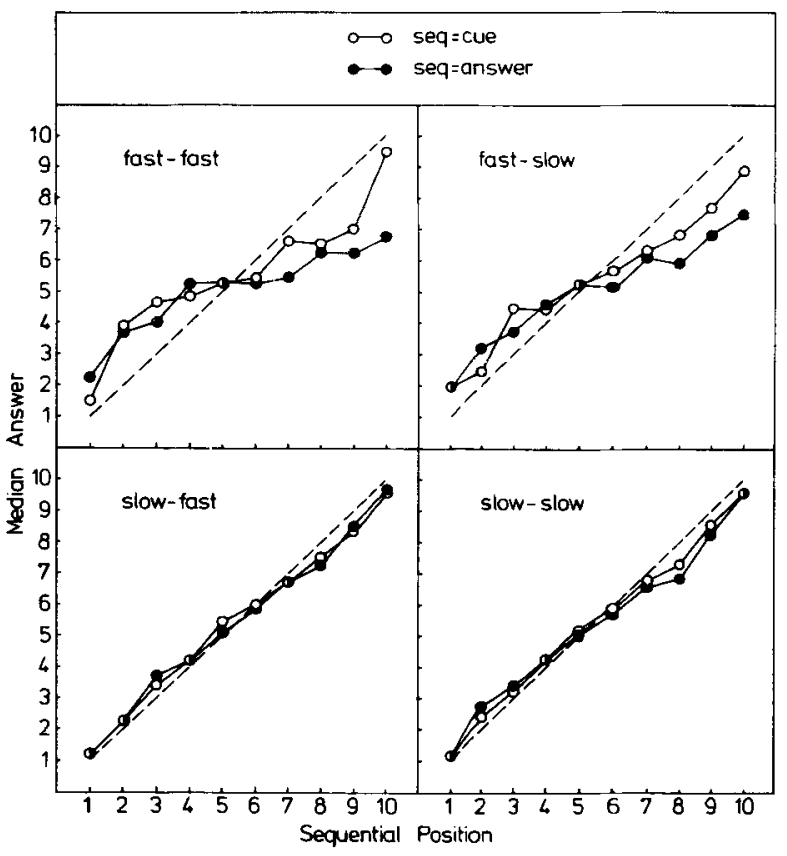

Figure 2. Median recalled values in Experiment 1 when sequential position was cue $(\mathrm{seq}=\mathrm{cue})$ or answer $(\mathrm{seq}=$ answer), for different rates of presentation (first) and recall (second). 


\section{Discussion}

The results of the present experiment enable progress (1) be made in locating the origin of the TAE reported by Jones (1976). The dependency of the effect upon speed of presentation suggests that it originates at the encoding stage rather than at retrieval itself. The effect's independence of speed of recall is consistent with this, although the null result may of course result from an inadequate range of variation in the condition. The approximately power-function relationship between the median recalled sequential position and its correct value, for fast presentation, is consistent with the representation of sequential position as the displacement of an item from the beginning of its list. The two values estimated for the power-law exponent, .44 and .55 for the fast-fast and fast-slow conditions, respectively, were approximately equal to each other and also consistent with the value of .44 found (under different conditions) by Jones (1976).

\section{EXPERIMENT 2}

The identification of the encoding stage as that which determines whether the TAE occurs carries the implication that the effect should be observed not only in delayed recall but also in immediate recall. It also suggests the further, stronger hypothesis that, if the conditions of encoding are the same, then the size of the effect should be the same for immediate and for delayed recall. These hypotheses were tested by an experiment which followed the design of the previous experiment except that the filled delay between presentation and recall was omitted.

\section{Method}

The method of this experiment was similar to that of the fast-fast condition of Experiment 1 . There were 20 subjects. The procedure differed in that after each list of words, the three-figure number from which to count backward by threes was omitted and replaced by the series of auditory bleeps signaling recall.

\section{Results}

Figure 3 shows the variation in the probability of correct recall in this experiment. The occurrence of a TAE is qualitatively apparent. A repeated-measures ANOVA, carried out as before upon arc-sine transformations of the proportions correct averaged over the first and second halves of the list, showed that the only significant factor was the interaction between type of cue and sequential position $[\mathrm{F}(1,19)=5.29, \mathrm{p}<.05]$.

The size of the TAE, measured in the same way as in Experiment 1, was 35 percentile points. This was approximately equal to the magnitudes observed in Experiment 1 (32 for fast-fast and 33 for slow-fast, respectively). The results of an ANOVA carried out (using the same procedures as before) upon the combined data of the present experiment and of the

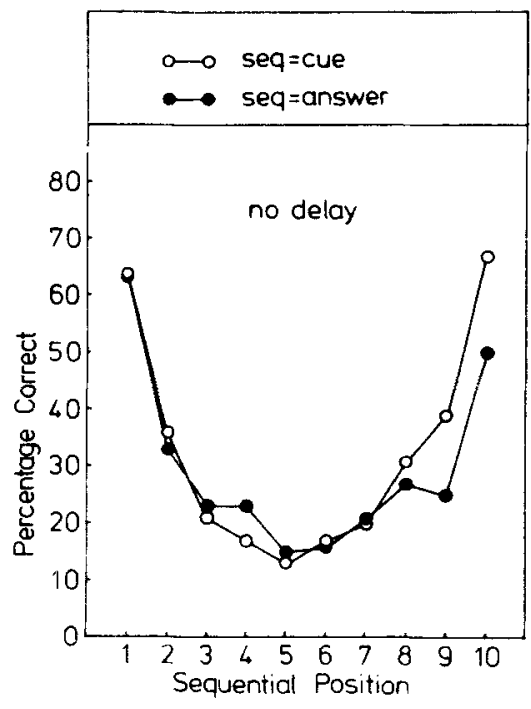

Figure 3. Percentage correct recall in Experiment 2 when sequential position was cue $(\mathrm{seq}=\mathrm{cue})$ or answer $(\mathrm{seq}=$ answer $)$.

fast-fast condition of Experiment 1 confirmed that the presence or absence of a delay period did not significantly affect the magnitude of the TAE. Though the main effect of the delay period was significant $[F(1,38)=16.02, p<.001]$, the delay period did not significantly interact with type of cue and sequential position $[F(1,38)=.03]$ or with any other factors.

\section{GENERAL DISCUSSION}

The observation of a TAE of approximately unchanged magnitude in Experiment 2 was in agreement with the implication of Experiment 1 that holding the conditions of presentation constant should produce an approximately constant TAE for immediate and delayed recall. This result also runs counter to a prediction made by Bjork and Whitten (1974). Bjork and Whitten postulated that the serial position curve observed in long-term recall has its basis in retrieval, and predicted that the magnitude of a recency effect should diminish with increasing delay between presentation and recall. The approximately constant TAE obtained here suggests that it is not the time between presentation and recall of an item which is important but, instead, the time between the presentation of an item and the start or end of its list. However, caution should be exercised since it could be argued that the sizes of the TAE in Experiments 1 and 2 are not in fact directly comparable because of the higher level of recall overall in Experiment 2.

The results reported here indicate that a purely retrieval explanation of the TAE is inadequate. Two specific forms of the converse general encoding hypothesis were considered in the introduction, and one of these, the dual-component hypothesis, was shown 
to account for the original results of Jones (1976). The dual-component hypothesis postulates that information about sequential position may be encoded in two different forms, relating either to the beginning or to the end of the list. The hypothesis is consistent with the present results if it is assumed that, at slow presentation rates, subjects have time to synthesize a single consistent representation of sequential position instead of the two conflicting representations otherwise encoded. It must remain speculative, however, until more direct evidence is obtained concerning the possibility of the multiple representation of sequential position in memory. At present it is possible to conclude only that the origins of the TAE are at the encoding stage.

\section{REFERENCES}

Asch. S. E.. \& Ebenholtz, S. M. The principle of associative symmetry. Proceedings of the American Philosophical Society. 1962. 106. 135-163.

BjoRK. R. A.. \& WhitTen. W. B. Recency-sensitive retrieval processes in long-term free recall. Cognitive Psychology. 19-4. 6, 173-189.

Horowitz, L. M., Norman, S. A.. \& Day. R. A. Arailability and associative symmetry. Psychological Review. 1966. 73, 1-15.

JOHNSON. D. M. The psychology of thought and judgment. New York: Harper. 1955.

JONES. G. V. A fragmentation hypothesis of memory: Cued recall of pictures and of sequential position. Journal of Experimental Psychology: General, 1976, 105, 277-293.

JoNes, G. V. Analyzing memory by cuing. In $\mathbf{N} . S$. Sutherland (Ed.). Tutorial essays in psychology. Potomac: Erlbaum. in press.

Lockhart, R. A. Retrieval asymmetry in the recall of adjectives and nouns. Journal of Experimental Psychology, 1969, 79, 12-17.

LowRY. D. H., \& WOLlen, K. A. Immediate and twentyfour hour retention of $\mathrm{S}-\mathrm{R}$ and $\mathrm{R}-\mathrm{S}$ associations. Journal of Experimental Psychology, 1969, 79, 59-62.

Paivio, A.. Yuille, J. C., \& Madigan, S. A. Concreteness, imagery and meaningfulness values for 925 nouns. Journal of Experimental Psychology Monograph. 1968. 76( 1, Part 2).

Peterson. L. R.. \& Peterson, M. J. Short-term retention of individual verbal items. Joumal of Experimental Psychology, 1959. 58, 193-198.

Peterson. M. J. Inagery and the grammatical classification of cues. Journal of Experimental Psychology, 1971, 88, 307-313.

REED. A. V. Speed-accuracy tradeoff in recognition memory. Science. 1973, 181. 574-576

Stevens. S. S. Issues in psychophysical measurement. Psichological Revieni, 1971, 78. 426-450.

Wickelgren. W. A. Network strength theory of storage and retrieval dynamics. Psychological Review. $1976,83$. 466-478.

Wolford. G. Function of distinct associations for pairedassociate performance. Psychological Review. 1971. 78. 303-313.

(Received for publication January 18, 1977; revision accepted June 24,1977 .) 